\title{
Portal Hypertensive Gastropathy in Chronic Hepatitis C Patients with Bridging Fibrosis and Compensated Cirrhosis: Results from the HALT-C Trial
}

Robert J. Fontana, M.D., ${ }^{1}$ Arun J. Sanyal, M.D., ${ }^{2}$ Savant Mehta, M.D., ${ }^{3}$ Michael C. Doherty, M.S., ${ }^{4}$ Brent A. Neuschwander-Tetri, M.D., ${ }^{5}$ Gregory T. Everson, M.D., ${ }^{6}$ Jeffrey A. Kahn, M.D., ${ }^{7}$ Peter F. Malet, M.D., ${ }^{8}$ Muhammad Y. Sheikh, M.D., ${ }^{9}$ Raymond T. Chung, M.D., ${ }^{10}$ Marc G. Ghany, M.D., ${ }^{11}$ David R. Gretch, M.D., ${ }^{12}$ and the HALT-C Trial Group

${ }^{1}$ Division of Gastroenterology, University of Michigan Medical Center, Ann Arbor, Michigan, ${ }^{2}$ Division of Gastroenterology, Virginia Commonwealth University Health System, Richmond, Virginia, ${ }^{3}$ Gastroenterology Division, University of Massachusetts Medical Center, Worcester, Massachusetts, ${ }^{4}$ New England Research Institutes, Watertown, Massachusetts, ${ }^{5}$ Division of Gastroenterology and Hepatology, Saint Louis University School of Medicine, St. Louis, Missouri, ${ }^{6}$ Section of Hepatology, Division of Gastroenterology and Hepatology, University of Colorado School of Medicine, Denver, Colorado, ${ }^{7}$ Division of Gastrointestinal and Liver Diseases, Keck School of Medicine, University of Southern California, Los Angeles, California, ${ }^{8}$ Division of Digestive and Liver Diseases, University of Texas Southwestern Medical Center, Dallas, Texas, ${ }^{9}$ Division of Gastroenterology, University of California -Irvine, Irvine, California, ${ }^{10}$ Gastrointestinal Unit (Medical Services), Massachusetts General Hospital and the Department of Medicine, Harvard Medical School, Boston, Massachusetts, ${ }^{11}$ Liver Diseases Branch, Division of Digestive Diseases and Nutrition, National Institute of Diabetes and Digestive and Kidney Diseases, National Institutes of Health, Department of Health and Human Services, Bethesda, Maryland, and ${ }^{12}$ Departments of Laboratory Medicine and Medicine, University of Washington, Seattle, Washington

OBJECTIVES: The clinical significance of portal hypertensive gastropathy (PHG) in patients with compensated liver disease is not well established. The aim of this study was to determine the prevalence and correlates of PHG in a large cohort of patients with chronic hepatitis C virus (HCV) infection and bridging fibrosis/compensated cirrhosis entering the randomized phase of the Hepatitis C Antiviral Long-term Treatment against Cirrhosis trial (HALT-C).

METHODS: $\quad$ The presence and severity of PHG in 1,016 HCV patients with no prior history of gastrointestinal bleeding was determined at surveillance endoscopy using the New Italian Endoscopy Club criteria.

RESULTS: $\quad$ Overall, $37 \%$ of HALT-C patients had PHG with $34 \%$ having mild and $3 \%$ with severe changes. The mucosal mosaic pattern was identified in 33\%, red marks in $15 \%$, and gastric antral vascular ectasia (GAVE) features in only 3\%. Independent correlates of PHG included biochemical markers of liver disease severity (lower serum albumin, higher bilirubin), portal hypertension (lower platelet count), insulin resistance (higher glucose), and non-African American race. Independent correlates of GAVE included a history of smoking, nonsteroidal anti-inflammatory drugs (NSAIDs) use within the past year, and higher serum bilirubin and glucose levels. There was a strong positive association between the presence of PHG and esophageal varices $(p<0.0001)$.

CONCLUSIONS: PHG is associated with the histological and biochemical severity of liver disease in patients with HCV and advanced fibrosis but is mild in most patients. The clinical relevance of these findings will be further explored during the randomized phase of the HALT-C study.

(Am J Gastroenterol 2006;101:983-992)

\section{INTRODUCTION}

Portal hypertensive gastropathy (PHG) is an endoscopic abnormality of the gastric mucosa that is most commonly ob-

This is publication \#12 from the HALT-C Trial Group. served in patients with advanced liver disease and/or portal hypertension $(1,2)$. This endoscopic lesion is characterized by the presence of a mucosal mosaic pattern with a snake skin-like appearance, focal red and brown spots, and vascular ectasias localized to the fundus or body of the stomach 
(2-5). The severity of PHG is commonly graded as mild or severe but the condition is very dynamic and some patients spontaneously improve while others worsen despite no apparent change in liver disease status (4-7). Ligation of varices and sclerotherapy have been associated with the development of de novo PHG in up to $50 \%$ of patients during short-term follow-up $(8,9)$. Patients with PHG are at increased risk of acute as well as chronic gastrointestinal bleeding although the annual incidence of bleeding has been difficult to quantify and distinguish from that due to esophagogastric varices $(6,7,10)$.

Prior studies have suggested that PHG is more common in patients with decompensated cirrhosis compared to those with compensated liver disease $(2,6,11)$. However, most of these studies were of limited size and included patients with varying etiologies of liver disease $(2,6)$. The aim of the current study was to determine the prevalence of PHG in a large cohort of well-characterized patients with chronic hepatitis $\mathrm{C}$ and biopsy proven bridging fibrosis or cirrhosis who were enrolled in the Hepatitis C Antiviral Longterm Treatment against Cirrhosis trial (HALT-C). In addition, we set out to identify baseline laboratory and clinical features associated with the presence and severity of PHG. Finally, we determined the relationship between esophageal varices and PHG in this large cohort of patients without a history of prior gastrointestinal bleeding or endoscopic therapy.

\section{METHODS}

\section{Patient Population}

This study included all patients entering the randomized phase of the HALT-C trial who underwent a screening esophagogastroduodenoscopy (EGD). Before enrollment, all subjects signed a written informed consent that had been approved by the local Institutional Review Boards at each of the 10 participating sites (12). The data were analyzed at the data coordinating center (New England Research Institute, Boston, MA). The entry and exclusion criteria for the HALT-C trial have been previously published $(12,13)$. Briefly, all HALT-C patients had detectable hepatitis $\mathrm{C}$ virus (HCV) RNA, a liver biopsy within 12 months of entry demonstrating an Ishak fibrosis score of 3 or 4 (bridging fibrosis) or 5-6 (cirrhosis), and nonresponse to prior interferon therapy. Subjects with a Child-Turcotte-Pugh score of $>6$ or a prior history of variceal/portal hypertensive bleeding, ascites, or hepatic encephalopathy were excluded. Enrolled subjects were retreated with pegylated Interferon $\alpha 2 \mathrm{a}(180 \mu \mathrm{g} / \mathrm{wk}$; Pegasys $^{\mathrm{TM}}$, Roche Laboratories, Nutley NJ) and ribavirin (1,000-1,200 mg daily, Copegus ${ }^{\mathrm{TM}}$, Roche). Those with persistently detectable HCV RNA at week 20 were eligible for randomization to either pegylated Interferon $\alpha 2 \mathrm{a} 90 \mu \mathrm{g} / \mathrm{wk}$ or observation without therapy for the next $3.5 \mathrm{yr}$. Week 20 virologic responders received a full 48 -wk course of peginterferon and ribavirin. Patients with virologic breakthrough during treatment or those with relapse after week 48 were also eligible for randomization. In addition, subjects with Ishak 3-6 fibrosis who had failed to respond to at least $24 \mathrm{wk}$ of pegylated interferon and ribavirin given outside of the trial, referred to as "Express" patients, were eligible for randomization.

A pretreatment medical history and physical exam was performed before enrollment. Lifetime alcohol consumption was assessed using a modified Skinner survey (14). All liver biopsies were evaluated by a central group of pathologists and the degree of hepatic fibrosis and inflammation were defined using the Ishak scoring system (15). Fasting insulin and glucose levels were obtained in all patients except the Express group. Insulin resistance was determined by the homeostatic model ( $\left.\mathrm{IR}_{\mathrm{HOMA}}\right)$ (16). Splenomegaly was defined as a spleen length $>13 \mathrm{~cm}$ on sonography.

\section{Endoscopy}

The protocol for EGD including the criteria for PHG grading was written in a manual of procedures and members at all centers were trained on the PHG scoring system before study initiation. The presence and severity of PHG were scored by the local study endoscopist according to the New Italian Endoscopy Conference criteria (NIEC) on a standard form (4). Specifically, the mucosal mosaic pattern was considered mild and scored 1 if there were diffuse pink polygonal, scalelike areas and severe with a score of 2 if present throughout the mucosa. Focal flat or slightly bulging red marks were considered mild and scored 1 if found in isolated areas and severe with a score of 2 if present throughout the mucosa. Gastric antral vascular ectasia (GAVE) was diagnosed by the presence of flat or slightly raised, red stripe-like lesions radiating from the pylorus to the antrum and body of the stomach and given a score of 2 . The presence of black-brown spots representative of old submucosal hemorrhage were not recorded. A PHG score of 1-3 was considered mild PHG while a score of 4 or more was considered severe PHG (4). The presence and size of esophageal varices were graded as small (F1) when they impinged on $25 \%$ of the lumen, medium (F2) when between F1 and F3, and large (F3) if the varices impinged on $>50 \%$ of the lumen. Other findings such as the presence of a hiatal hernia, gastric ulcer, and duodenal ulcer were reported in a text field by the local endoscopist.

\section{Statistical Analysis}

Continuous variables with non-normal distribution were log transformed. Univariate analysis of demographic, liver disease, and clinical factors as correlates of the presence and severity of PHG and its component features were undertaken. In addition, multivariate logistic regression analysis with stepwise selection of significant independent predictors was performed to identify independent correlates of the presence and severity of PHG as well as GAVE. 


\section{RESULTS}

\section{Patient Population}

A total of 1,050 subjects were enrolled in the randomized phase of the HALT-C trial of whom 1,016 (97\%) underwent EGD and were included in this study (Table 1). The mean age of participants was $50.2 \mathrm{yr}$, there was a preponderance of men (71\%), and 18\% were African American. The majority of subjects were abstinent from alcohol (86\%) and cigarettes $(70 \%)$ at enrollment. Forty-nine percent of subjects reported use of nonsteroidal anti-inflammatory drugs (NSAIDs) during the 12 months prior to their endoscopy while only $14 \%$ were receiving a proton pump inhibitor (PPI) and 5\% a cyclo- oxygenase type 2 (COX-2) inhibitor at the time of EGD. As expected, there was an overrepresentation of HCV genotype 1 in this group of nonresponders to prior pegylated interferon and ribavirin. The mean body mass index (BMI) was 29.9 $\mathrm{kg} / \mathrm{m}^{2}$ and $24 \%$ of subjects were diabetic.

There were 598 subjects $(58 \%)$ with Ishak stage 3 or 4 fibrosis and 418 subjects (42\%) with Ishak stage 5 or 6 fibrosis. The proportion of patients with diabetes mellitus, Mallory bodies on biopsy, and the mean Ishak inflammatory scores were significantly higher in subjects with cirrhosis compared to those with bridging fibrosis. Conversely, subjects with bridging fibrosis had significantly higher platelet counts and albumin levels and lower serum bilirubin, alanine

Table 1. Clinical Features of the HALT-C Study Population

\begin{tabular}{|c|c|c|c|c|}
\hline Parameter & All Subjects $N=1,016$ & Ishak 3 or $4 \mathrm{~N}=598$ & Ishak 5 or $6 \mathrm{~N}=418$ & Ishak 3/4 vs 5/6p Value* \\
\hline Age (yr) & $50.2+7.2$ & $50.2+7.3$ & $50.2+7.0$ & 0.84 \\
\hline Females $(\%)$ & 29 & 30 & 28 & 0.43 \\
\hline African American (\%) & 18 & 20 & 16 & 0.046 \\
\hline Lead-in (\%) & 63 & 61 & 65 & 0.08 \\
\hline Breakthrough/relapse (\%) & 14 & 17 & 11 & \\
\hline Express $(\%)$ & 23 & 22 & 24 & \\
\hline No alcohol for 6 months (\%) & 86 & 84 & 89 & 0.027 \\
\hline Lifetime alcohol/day (g/day) & $25.8+40.2$ & $24.9+39.4$ & $26.9+41.4$ & 0.44 \\
\hline No smoking for 6 months (\%) & 70 & 71 & 70 & 0.65 \\
\hline Lifetime smoking (pack yr) & $14.8+16.7$ & $15.1+16.8$ & $14.5+16.6$ & 0.61 \\
\hline Splenomegaly $(\%)$ & 33 & 24 & 47 & $<0.001$ \\
\hline Body mass index $\left(\mathrm{kg} / \mathrm{m}^{2}\right)$ & $29.9+5.5$ & $29.7+5.6$ & $30.3+5.3$ & 0.064 \\
\hline Diabetes mellitus (\%) & 24 & 22 & 28 & 0.019 \\
\hline Hypertension $(\%)$ & 35 & 33 & 37 & 0.27 \\
\hline $\log _{10} \operatorname{IR}_{\mathrm{HOMA}}{ }^{\dagger}$ & $2.28+0.92$ & $2.18+0.94$ & $2.43+0.88$ & 0.0002 \\
\hline $\log _{10} \operatorname{IR}_{\text {HOMA(nondiabetic) }}{ }^{\dagger}$ & $2.01+0.75$ & $1.93+0.78$ & $2.14+0.68$ & 0.001 \\
\hline $\log _{10}$ glucose & $4.65+0.3$ & $4.63+0.29$ & $4.68+0.3$ & 0.0164 \\
\hline $\log _{10}$ glucose (nondiabetic) & $4.53+0.14$ & $4.52+0.15$ & $4.54+0.13$ & 0.121 \\
\hline $\log _{10}$ insulin & $3.63+0.75$ & $3.55+0.77$ & $3.75+0.70$ & 0.0002 \\
\hline $\log _{10}$ insulin (nondiabetic) & $3.49+0.69$ & $3.42+0.71$ & $3.60+0.64$ & 0.0014 \\
\hline $\log _{10}$ HCV RNA & $14.8+1.2$ & $15.0+1.2$ & $14.5+1.3$ & $<0.0001$ \\
\hline HCV genotype $1(\%)$ & 94 & 94 & 93 & 0.28 \\
\hline Duration of infection (yr) & $28+8$ & $28+8$ & $29+8$ & 0.086 \\
\hline Ishak inflammation & $7.5+2.0$ & $7.2+2.0$ & $8.0+2.0$ & $<0.001$ \\
\hline Mallory bodies (\%) & 15 & 11 & 22 & $<0.001$ \\
\hline Hemoglobin (g/dL) & $15.0+1.4$ & $15.1+1.5$ & $14.9+1.4$ & 0.022 \\
\hline Platelets $\left(10^{3} / \mathrm{mL}\right)$ & $165+66$ & $187+62$ & $134+55$ & $<0.001$ \\
\hline Iron saturation $(\%)$ & $36+16$ & $38+18$ & $35+15$ & 0.0014 \\
\hline $\log _{10}$ ferritin $(\mathrm{ng} / \mathrm{mL})$ & $2.36+0.47$ & $2.36+0.47$ & $2.35+0.48$ & 0.89 \\
\hline Serum AST $(\times$ ULN) & $2.1+1.5$ & $1.9+1.3$ & $2.4+1.6$ & $<0.001$ \\
\hline Serum ALT $(\times$ ULN $)$ & $2.1+1.6$ & $2.0+1.6$ & $2.3+1.6$ & 0.020 \\
\hline AST: ALT ratio & $0.88+0.29$ & $0.84+0.28$ & $0.94+0.31$ & $<0.001$ \\
\hline Alkaline phosphatase $(\times$ ULN $)$ & $0.85+0.42$ & $0.80+0.42$ & $0.93+0.42$ & $<0.001$ \\
\hline Bilirubin (mg/dL) & $0.79+0.40$ & $0.72+0.35$ & $0.89+0.45$ & $<0.001$ \\
\hline INR & $1.04+0.11$ & $1.01+0.10$ & $1.08+0.10$ & $<0.001$ \\
\hline Albumin $(\mathrm{g} / \mathrm{dL})$ & $3.87+0.40$ & $3.95+0.35$ & $3.76+0.43$ & $<0.001$ \\
\hline Biopsy to EGD (days) & $287+192$ & $302+197$ & $265+183$ & 0.0024 \\
\hline NSAID at EGD (\%) & 9 & 10 & 9 & 0.70 \\
\hline NSAID within 12 months (\%) & 49 & 50 & 47 & 0.39 \\
\hline COX-2 at EGD $(\%)$ & 5 & 5 & 5 & 0.72 \\
\hline Beta-blocker at EGD (\%) & 9 & 9 & 10 & 0.41 \\
\hline PPI at EGD $(\%)$ & 14 & 14 & 14 & 0.92 \\
\hline
\end{tabular}

Data presented as mean \pm SD or $(\%)$.

${ }^{*} T$-tests were used to compare means and $\chi^{2}$ for proportions of patients with Ishak $3 / 4 v s$ Ishak 5/6.

${ }^{\dagger}$ Tested in only 776 non express patients. 


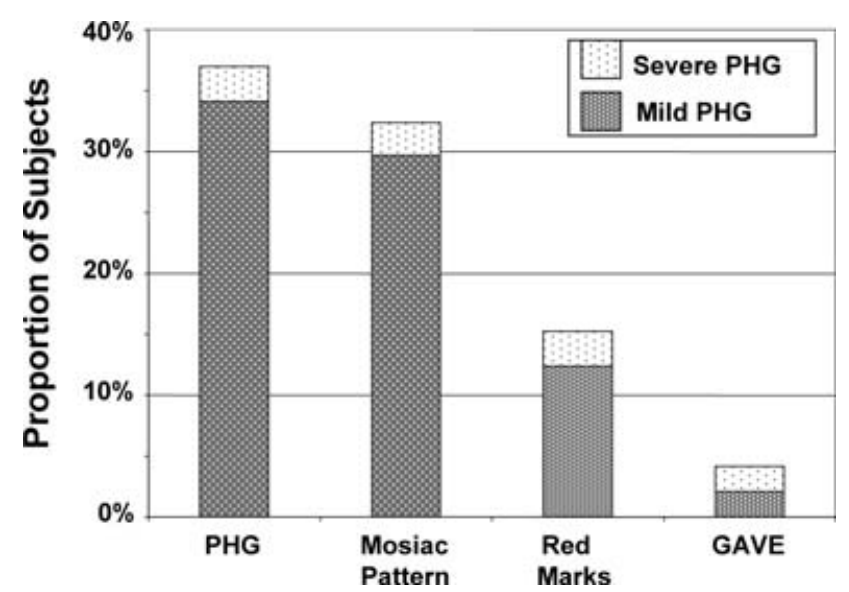

Figure 1. Prevalence of mild and severe portal hypertensive gastropathy $(\mathrm{PHG})$ and component features in HALT-C patients.

and aspartate aminotransferase (ALT, AST), alkaline phosphate, and INR levels compared to subjects with cirrhosis.

\section{Prevalence of $P H G$}

Overall, 374 subjects (37\%) had PHG identified at surveillance EGD. In the majority of cases, PHG was mild (34\%) and only 29 patients (3\%) had severe changes. The most frequent endoscopic feature suggestive of PHG was the presence of mucosal mosaic pattern which was seen in $33 \%$ of subjects (Fig. 1). In contrast, isolated or confluent red marks were reported in $15 \%$ of subjects and GAVE features were noted in only $3 \%$. If GAVE features were present, the subject was more likely to have severe PHG compared to subjects with PHG and no GAVE lesions (odds ratio $=40.4(15.9,101.9)$ $p<0.0001)$.

Both mild and severe PHG were significantly associated with the stage of hepatic fibrosis (Fig. 2, $p<0.0001$ for trend). The mean levels of several biochemical markers of liver disease severity (i.e., albumin, bilirubin, and INR) were also significantly worse among subjects with PHG compared to those without PHG (Table 2). Similarly, the mean Ishak fibrosis scores and the prevalence of splenomegaly were sig-

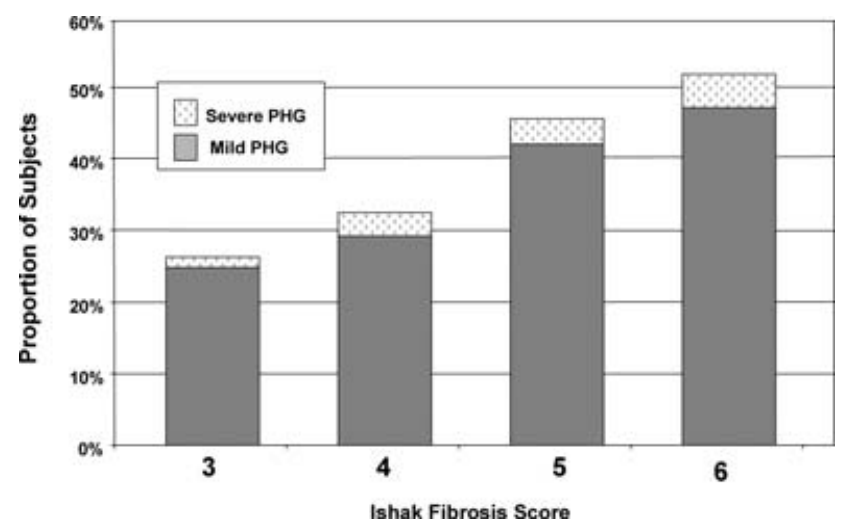

Figure 2. Proportion of patients with chronic hepatitis $C$ and Ishak fibrosis scores of 3-6 with mild or severe portal hypertensive gastropathy (PHG) $(p<0.0001$ for trend). nificantly higher among those with PHG compared to those without PHG. Interestingly, Mallory bodies were more frequently noted in subjects with PHG. In addition, the $\mathrm{IR}_{\mathrm{HOMA}}$ and glucose levels were significantly higher among subjects with PHG and these differences were most pronounced in nondiabetic patients. However, the prevalence of diabetes and mean BMI levels were not significantly greater in subjects with PHG compared to subjects without PHG. PHG was less frequently identified in African American patients (35\%) compared to others $(39 \%)(p=0.037)$.

Although we had anticipated that lifetime smoking and alcohol consumption would be associated with PHG, neither of these measures showed a significant association. In addition, use of NSAIDs, PPIs, COX-2 inhibitors, and beta-blockers at the time of EGD was not associated with PHG. Finally, the mean hemoglobin levels and iron stores of subjects with PHG were not significantly different from those without PHG.

\section{Correlates of PHG}

On univariate analysis, serum alkaline phosphatase, AST/ALT ratio, splenomegaly, INR, bilirubin, $\log _{10} \mathrm{IR}_{\mathrm{HOMA}}$, and $\log _{10}$ glucose levels were all associated with a greater likelihood of PHG. In contrast, African American race, serum albumin, and platelet counts were associated with a lower frequency of PHG. Using stepwise logistic regression, non-African American race, albumin, platelets, bilirubin, and $\log _{10}$ glucose were independently associated with PHG (Table 3 ). However, the overall model $c$-statistic was only 0.645 and the majority of the variance was accounted for by low platelet count. Models with similar predictive ability were obtained when assessing the presence of PHG in patients with bridging fibrosis and cirrhosis separately (data not shown). Positive correlates of severe PHG in the overall cohort included AST/ALT ratio, bilirubin, Ishak fibrosis score, and $\log _{10}$ glucose levels while platelet counts were negatively associated with severe PHG. Using logistic regression analysis, only $\log _{10}$ glucose and total bilirubin levels were significant independent correlates of severe PHG (Table 3).

Since GAVE features are most commonly associated with gastrointestinal bleeding, models to predict these changes were derived. On univariate analysis, smoking history, bilirubin, Ishak fibrosis score, NSAID use in the past 12 months, and $\log _{10}$ glucose were positively associated with GAVE while platelet count and time from biopsy to endoscopy were negatively associated with GAVE. When using stepwise selection, we identified bilirubin, NSAID use, smoking history, and $\log _{10}$ glucose as independent correlates of GAVE. However, the prevalence of GAVE in the study population was low $(3 \%)$ and additional studies in patients with a higher prevalence of GAVE are needed to confirm the relatively weak associations noted.

\section{Esophageal Varices and PHG}

Previous studies have suggested a positive relationship between the presence of esophageal varices and PHG $(2,9)$. Studies of the HALT-C cohort have shown that $26 \%$ of 
Table 2. HALT-C Patients with and Without Portal Hypertensive Gastropathy

\begin{tabular}{|c|c|c|c|c|c|}
\hline Parameter & All Subject $N=1,016$ & No. PHG N $=637$ & PHG Present $\mathrm{N}=374$ & Odds Ratio* $(95 \% \mathrm{CI})$ & $p$ Value* \\
\hline Age (yr) & $50.2+7.2$ & $50.2+7.4$ & $50.2+6.6$ & $1.00(0.88,1.14)$ & 1.000 \\
\hline Females $(\%)$ & 29 & 30 & 28 & $0.87(0.66,1.16)$ & 0.352 \\
\hline African American (\%) & 18 & 21 & 15 & $0.68(0.48,0.96)$ & 0.027 \\
\hline Lead-in $(\%)$ & 63 & 62 & 64 & & 0.91 \\
\hline Breakthrough/relapse (\%) & 14 & 15 & 14 & & \\
\hline Express $(\%)$ & 23 & 23 & 22 & & \\
\hline No alcohol for 6 months (\%) & 86 & 87 & 84 & $0.82(0.57,1.17)$ & 0.272 \\
\hline Lifetime alcohol/day (g/day) & $25.8+40.2$ & $24.9+39.4$ & $26.9+41.4$ & $1.03(0.90,1.17)$ & 0.67 \\
\hline No smoking for 6 months (\%) & 70 & 72 & 68 & $1.21(0.91,1.59)$ & 0.19 \\
\hline Lifetime smoking (pack yr) & $14.8+16.7$ & $15.1+16.8$ & $14.5+16.6$ & $1.08(0.95,1.23)$ & 0.24 \\
\hline Splenomegaly (\%) & 33 & 30 & 39 & $1.45(1.10,1.90)$ & 0.0075 \\
\hline Body mass index $\left(\mathrm{kg} / \mathrm{m}^{2}\right)$ & $29.9+5.5$ & $29.7+5.6$ & $30.3+5.3$ & $1.03(0.91,1.17)$ & 0.64 \\
\hline Diabetes Mellitus (\%) & 24 & 23 & 27 & $1.27(0.94,1.70)$ & 0.12 \\
\hline Hypertension $(\%)$ & 35 & 34 & 35 & $1.05(0.80,1.37)$ & 0.73 \\
\hline $\log _{10} \operatorname{IR}_{\mathrm{HOMA}}^{\dagger}$ & $2.28+0.92$ & $2.23+0.91$ & $2.38+0.93$ & $1.18(1.02,1.37)$ & 0.025 \\
\hline $\log _{10} \operatorname{IR}_{\text {HOMA(nondiabetic) }}^{\dagger}$ & $2.01+0.75$ & $1.97+0.76$ & $2.08+0.72$ & $1.16(0.98,1.37)$ & 0.297 \\
\hline $\log _{10}$ glucose & $4.65+0.30$ & $4.63+0.30$ & $4.68+0.3$ & $1.18(1.04,1.34)$ & 0.01 \\
\hline $\log _{10}$ glucose (nondiabetic) & $4.53+0.14$ & $4.52+0.15$ & $4.55+0.13$ & $1.21(1.04,1.41)$ & 0.0095 \\
\hline $\log _{10}$ insulin & $3.63+0.75$ & $3.59+0.74$ & $3.70+0.75$ & $1.15(0.99,1.33)$ & 0.060 \\
\hline $\log _{10}$ insulin (nondiabetic) & $3.49+0.69$ & $3.46+0.70$ & $3.54+0.67$ & $1.14(0.96,1.35)$ & 0.14 \\
\hline $\log _{10}$ HCV RNA & $14.8+1.2$ & $15+1.1$ & $14.5+1.3$ & $0.89(0.79,1.01)$ & 0.071 \\
\hline HCV genotype $1(\%)$ & 94 & 94 & 93 & $0.88(0.52,1.48)$ & 0.63 \\
\hline Duration of infection (yr) & $28.1+8.0$ & $27.7+8.0$ & $28.6+8.1$ & $1.05(0.92,1.20)$ & 0.44 \\
\hline Ishak fibrosis score & $4.1+1.2$ & $3.9+1.2$ & $4.4+1.3$ & $1.40(1.26,1.56)$ & $<0.0001$ \\
\hline Ishak inflammation & $7.5+2.0$ & $7.2+2.0$ & $8.0+2.0$ & $1.19(1.05,1.36)$ & 0.006 \\
\hline Mallory bodies (\%) & 15 & 12 & 20 & $1.77(1.25,2.50)$ & 0.0011 \\
\hline Hemoglobin (g/dL) & $15.0+1.4$ & $15.1+1.5$ & $14.9+1.4$ & $1.03(0.91,1.18)$ & 0.62 \\
\hline Platelets $\left(10^{3} / \mathrm{mL}\right)$ & $164+65$ & $186+63$ & $134+57$ & $0.64(0.55,0.74)$ & $<0.0001$ \\
\hline Iron saturation (\%) & $36+16$ & $38+18$ & $35+15$ & $1.07(0.95,1.22)$ & 0.29 \\
\hline $\log _{10}$ ferritin $(\mathrm{mg} / \mathrm{dL})$ & $2.36+0.47$ & $2.36+0.48$ & $2.35+0.45$ & $0.99(0.87,1.12)$ & 0.84 \\
\hline Serum AST $(\times$ ULN) & $2.1+1.5$ & $1.87+1.33$ & $2.43+1.64$ & $1.13(0,99,1.29)$ & 0.061 \\
\hline Serum ALT $(\times$ ULN $)$ & $2.14+1.63$ & $2.04+1.64$ & $2.28+1.61$ & $1.04(0.92,1.18)$ & 0.53 \\
\hline AST: ALT ratio & $0.88+0.29$ & $0.84+0.28$ & $0.94+0.42$ & $1.25(1.10,1.42)$ & 0.0005 \\
\hline Alkaline phosphatase $(\times$ ULN $)$ & $0.85+0.42$ & $0.80+0.42$ & $0.93+0.42$ & $1.19(1.05,1.36)$ & 0.0069 \\
\hline Bilirubin (mg/dL) & $0.79+0.4$ & $0.72+0.35$ & $0.89+0.42$ & $1.40(1.23,1.60)$ & $<0.0001$ \\
\hline INR & $1.04+0.11$ & $1.01+0.10$ & $1.08+0.10$ & $1.40(1.22,1.60)$ & $<0.0001$ \\
\hline Albumin (g/dL) & $3.87+0.4$ & $3.95+0.4$ & $3.8+0.4$ & $0.72(0.63,0.82)$ & $<0.0001$ \\
\hline Biopsy to EGD (days) & $287+192$ & $302+197$ & $265+183$ & $1.00(0.88,1.14)$ & 0.94 \\
\hline NSAID at EGD (\%) & 9 & 9 & 10 & $1.09(0.70,1.70)$ & 0.71 \\
\hline NSAID within 12 months (\%) & 49 & 51 & 46 & $0.82(0.63,1.06)$ & 0.13 \\
\hline COX-2 at EGD $(\%)$ & 5 & 5 & 5 & $1.06(0.58,1.93)$ & 0.86 \\
\hline Beta-blocker at EGD (\%) & 9 & 8 & 11 & $1.47(0.94,2.28)$ & 0.087 \\
\hline PPI at EGD (\%) & 14 & 15 & 14 & $0.93(0.63,1.36)$ & 0.69 \\
\hline
\end{tabular}

Data presented as mean + SD or $(\%)$.

$\mathrm{PPI}=$ proton pump inhibitor; $\mathrm{PHG}=$ portal hypertensive gastropathy.

${ }^{*}$ Comparison of subjects with and without portal hypertensive gastropathy.

${ }^{\dagger}$ Tested in only 776 nonexpress patients.

patients had esophageal varices and that varices were significantly more common in subjects with cirrhosis compared to those with bridging fibrosis (39\% vs $16 \%, p<0.0001)$ (17). For these reasons, the positive association between the presence of PHG and esophageal varices was not unexpected (Fig. 3). A total of $40 \%$ of patients with PHG had varices compared to only $17 \%$ of subjects without PHG ( $p$ $<0.0001)$. In addition, subjects with PHG were significantly more likely to have medium or large varices compared to subjects without PHG (odds ratio $=6.40(95 \% \mathrm{CI}: 3.47,11.79), p$ $<0.0001)$.

\section{Additional Endoscopic Findings}

A hiatal hernia was identified in 162 subjects (16\%), a gastric ulcer was noted in 18 subjects $(1.8 \%)$, a duodenal ulcer in 16 (1.6\%), and 5 of these subjects had both a gastric and a duodenal ulcer. There was a trend toward PHG being associated with the presence of a gastric ulcer and duodenal ulcer but neither trend was statistically significant. However, drinking alcohol in the past 6 months was positively associated with the presence of a gastric ulcer $(p=0.016)$. Similarly, drinking alcohol in the past 6 months $(p=0.043)$, NSAID use in the past 12 months $(p=0.035)$, and the presence of 
Table 3. Logistic Regression Models to Predict Portal Hypertensive Gastropathy, Severe Portal Hypertensive Gastropathy, and Gastric Antral Vascular Ectasia

\begin{tabular}{|c|c|c|c|c|}
\hline Variable & Estimate & Odds Ratio $(95 \% \mathrm{CI})$ & $p$ Value & $c$ Statistic \\
\hline \multicolumn{5}{|l|}{ Portal hypertensive gastropathy } \\
\hline African American & -0.434 & $0.65(0.45,0.93)$ & 0.020 & \multirow[t]{5}{*}{0.645} \\
\hline Platelets & -0.00407 & $0.99(0.99,0.998)$ & 0.0007 & \\
\hline Bilirubin & 0.570 & $1.77(1.24,2.51)$ & 0.0015 & \\
\hline Albumin & -0.627 & $0.53(0.37,0.76)$ & 0.0005 & \\
\hline $\log _{10}$ glucose & 0.548 & $1.73(1.10,2.71)$ & 0.017 & \\
\hline \multicolumn{5}{|c|}{ Severe portal hypertensive gastropathy } \\
\hline Bilirubin & 1.01 & $2.75(1.40,5.37)$ & 0.0031 & \multirow[t]{2}{*}{0.678} \\
\hline $\log _{10}$ glucose & 1.67 & $5.29(2.07,13.6)$ & 0.0005 & \\
\hline \multicolumn{5}{|l|}{ Gastric antral vascular ectasia } \\
\hline Bilirubin & 0.947 & $2.58(1.36,4.88)$ & 0.0037 & \multirow[t]{4}{*}{0.702} \\
\hline NSAID within 12 months & 0.703 & $2.02(1.04,3.90)$ & 0.036 & \\
\hline $\log _{10}$ glucose & 1.45 & $4.27(1.88,9.69)$ & 0.0005 & \\
\hline Ever smoked & 0.994 & $2.70(1.03,7.10)$ & 0.044 & \\
\hline
\end{tabular}

cirrhosis $(p=0.024)$ were all positively associated with duodenal ulcer.

\section{DISCUSSION}

The overall prevalence of PHG in this highly selected study population was $37 \%$, a proportion which is consistent with other cross-sectional studies involving patients with advanced but compensated chronic liver disease $(2,6)$. The lower frequency of severe compared to mild PHG is also consistent with prior studies $(2,6,18)$. The current study also demonstrated a strong positive association between the presence of PHG and esophageal varices (Fig. 3) (2, 9). The current study was unique in that it represents a large prospective cohort of well-characterized HCV patients who have undergone surveillance EGD. The presence and severity of PHG was graded in a standardized manner using a validated scoring system (18). In addition, all liver biopsies were reviewed by a central group of expert hepatopathologists and all patients underwent extensive laboratory and clinical assessment upon study entry. The prevalence and correlates of PHG from this study are likely to be representative of American patients with advanced but compensated HCV who have failed to respond to pegylated interferon and ribavirin.

The pathogenesis of PHG is not well understood but may be related to increased gastric mucosal blood flow mediated by activation of cytokines and growth factors $(11,19,20)$. There is no established link between Helicobacter pylori infection and the presence and severity of PHG $(21,22)$. Hemodynamic studies demonstrate that the severity of portal hypertension is related to the likelihood of having severe PHG but is not the sole determinant $(19,23)$. In particular, portal pressure measurements do not correlate with the presence or severity of GAVE and several authors have suggested alternative etiopathogenic mechanisms for $\operatorname{GAVE}(5,24,25)$. A recent study demonstrated that subjects with acute heavy alcohol ingestion were more likely to have gastroduodenal erosions and ulcers that were independently associated with the presence and severity of PHG (26). In the current study, however, no association was found between either lifetime or recent alcohol consumption and the prevalence or severity of PHG (Table 2). This lack of association may have been due to the fact that most of the HALT-C patients (86\%) were abstinent from alcohol at enrollment and the few who did consume alcohol were drinking minimally. Similarly, the current study also failed to demonstrate a relationship between NSAID and/or COX-2 inhibitor use and the presence or severity of PHG. Finally, although smoking has been associated with changes in gastric mucosal blood flow and mucosal ulceration, an association between PHG and smoking was not identified in this study cohort.

The expected associations between markers of liver disease severity and the presence and severity of PHG were identified on both univariate and multivariate analysis (Tables 2 and 3). In a logistic regression model, only non-African American race, low platelet count, high serum bilirubin, low serum

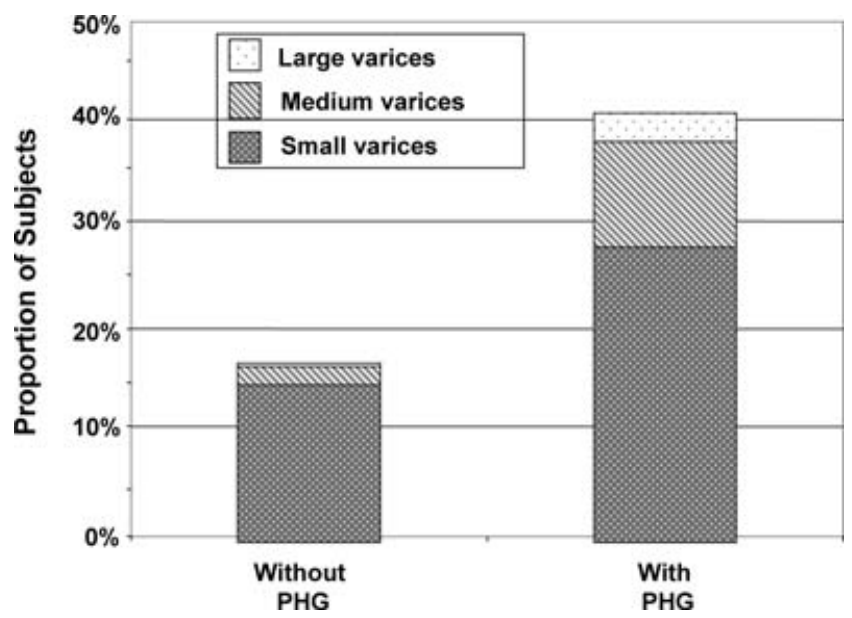

Figure 3. Prevalence of small, medium, and large esophageal varices in patients with and without portal hypertensive gastropathy (PHG) $(p<0.0001)$. 
albumin, and high glucose levels were found to be independent predictors of PHG. The reduced probability of African Americans having PHG is consistent with a prior analysis of this cohort in which African Americans were significantly less likely to have esophageal varices (17). The mechanism for a protective effect of race is not clear and the clinical significance is uncertain since PHG was identified in 35\% of African Americans and 39\% of non-African Americans. However, the prevalence of cirrhosis was lower $(35 \%$ vs $43 \%$, $p=0.0459)$ and the mean platelet counts were higher (180 vs $16110^{3} / \mathrm{mL}, p=0.0003$ ) among African American compared to non-African American HALT-C patients. The association between glucose levels and the presence and severity of PHG may relate to an increased frequency of glucose intolerance and insulin resistance among HCV patients with more severe hepatic fibrosis (27). Fibrotic remodeling of the liver can lead to reduced clearance of insulin and insulinlike growth factors with resultant splanchnic hyperinsulinemia that may stimulate hepatic stellate cells $(27,28)$. Several studies have also demonstrated increased rates of fibrosis progression among HCV patients with hepatic steatosis and insulin resistance $(29,30)$. Therefore, it is possible that the significant association between glucose levels and the presence and severity of PHG may reflect changes due to underlying insulin resistance but further physiologic studies are needed (31-34).

The prevalence of mild (3\%) and severe (1\%) GAVE was low in this highly selected patient population. This may in part be due to the predominance of men and the intentional exclusion of patients with a history of gastrointestinal bleeding or anemia at entry. In addition, only GAVE lesions localized to the antrum in a stripe-like appearance were scored while prior studies have suggested that vascular ectasias in patients with cirrhosis are more likely to be diffuse and located in the body $(24,35)$. On multivariate modeling, both increased serum bilirubin and glucose levels were positively associated with the presence of GAVE as were the recent use of an NSAID and a lifetime history of smoking. It is wellknown that NSAIDs can induce gastroduodenal erosions and ulcers and that NSAID gastropathy tends to localize to the antrum possibly due to motility effects (36). Interestingly, some studies have suggested that patients with cirrhosis and GAVE may display abnormal antral motility (37). Although GAVE lesions represent a distinct clinicopathological entity, the positive association of NSAID use with GAVE lesions in this patient population is consistent with prior work demonstrating reduced gastric mucosal gel thickness in patients with PHG and increased susceptibility to caustic agents $(38,39)$. Similarly, the well described epidemiological association between smoking and gastroduodenal ulceration may, in part, account for the positive association noted between smoking and GAVE (40-44).

Identifying risk factors for bleeding from PHG is difficult due to the low rate of spontaneous bleeding from this entity $(7,18)$. During longitudinal follow-up, subjects with severe
PHG are more likely to bleed than subjects with mild PHG $(6,18)$. In addition, $\mathrm{HCV}$ patients with cirrhosis and severe PHG were more likely to have a peptic ulcer compared to patients with mild PHG (22). In the current study, however, there was no significant relationship between baseline hemoglobin or iron levels with the presence or severity of PHG or the presence of GAVE lesions (data not shown). These observations may be due, in part, to the intentional exclusion of subjects with a prior history of gastrointestinal bleeding or anemia at entry. Further study and follow-up should demonstrate whether patients with PHG and/or GAVE features are more likely to develop gastrointestinal bleeding during the randomized phase of HALT-C.

Limitations of this study included the lack of central review of endoscopic photos for PHG grading (45). However, all endoscopists were trained on the scoring system and a standardized reporting form and manual of procedures were used at participating sites. The optimal means of scoring PHG remains controversial since few studies have demonstrated a relationship between PHG severity and the risk of gastrointestinal bleeding $(2,18,45)$. The PHG scoring system used in this study has been shown to have excellent interobserver agreement and to correlate with the risk of bleeding during short-term follow-up (18). However, as with other proposed PHG scoring systems, there is a lower interobserver agreement on scoring the severity of PHG using the NIEC criteria. Portal hemodynamic studies were not performed in this study because of the associated risks and resources required. However, prior studies that have attempted to correlate portal hemodynamic measurements with endoscopic findings have yielded inconsistent results $(11,19)$. Finally, subjects enrolled in HALT-C are not necessarily representative of HCV patients with advanced fibrosis in the general population since subjects with anemia, severe thrombocytopenia, or other contraindications to retreatment with pegylated IFN and ribavirin were excluded. In addition, subjects with a prior history of gastrointestinal bleeding, ascites, or encephalopathy were excluded. Nonetheless, this study represents the largest cohort of well-characterized American subjects with advanced but compensated HCV fibrosis who have undergone surveillance EGD.

In summary, $37 \%$ of $1,016 \mathrm{HCV}$ patients with bridging fibrosis or compensated cirrhosis had evidence of PHG but only 3\% had severe PHG. Independent correlates of PHG include biochemical markers of liver disease severity (low serum albumin, high bilirubin), portal hypertension (low platelet count), and markers of insulin resistance (high glucose levels). Subjects with a prior history of smoking and recent NSAID use were found to be at greatest risk of harboring GAVE but the low overall prevalence of this lesion limits the strength and implications of this finding. The clinical significance of PHG and GAVE as a source of gastrointestinal bleeding and as a potential correlate of disease progression will be tracked during the longitudinal phase of HALT-C. 


\section{ACKNOWLEDGMENTS}

This study was supported by the National Institute of Diabetes \& Digestive \& Kidney Diseases (contract numbers are listed below). Additional support was provided by the National Institute of Allergy and Infectious Diseases (NIAID), the National Cancer Institute, the National Center for Minority Health and Health Disparities, and by the General Clinical Research Center grants from the National Center for Research Resources, National Institutes of Health (grant numbers are listed below). Additional funding to conduct this study was supplied by Hoffmann-La Roche, Inc., through a Cooperative Research and Development Agreement (CRADA) with the National Institutes of Health.

In addition to the authors of this manuscript, the following individuals were instrumental in the planning, conduct and/or care of patients enrolled in this study at each of the participating institutions as follows:

University of Massachusetts Medical Center, Worcester, MA: (Contract N01-DK-9-2326) Dawn Bombard, R.N., Maureen Cormier, R.N., Donna Giansiracusa, R.N.

University of Connecticut Health Center, Farmington, CT: (Grant M01RR-06192) Herbert L. Bonkovsky, M.D., Michelle Kelley, R.N., A.N.P.

Saint Louis University School of Medicine, St Louis, MO: (Contract N01-DK-9-2324) Bruce Bacon, M.D., Adrian Di Bisceglie, M.D., Debra King, R.N., Judy Thompson, R.N.

Massachusetts General Hospital, Boston, MA: (Contract N01-DK-9-2319, Grant M01RR-01066), Jules L. Dienstag, M.D., Andrea E. Reid, M.D., Sarah Hanson, Jennifer Kittay.

University of Colorado School of Medicine, Denver, CO: (Contract N01-DK-9-2327, Grant M01RR-00051) Marcelo Kugelmas, M.D., Jennifer DeSanto, R.N., Carol McKinley, R.N., Brenda Easley, R.N.

University of California-Irvine, Irvine, CA: (Contract N01-DK-9-2320, Grant M01RR-00827) Timothy R. Morgan, M.D., John Hoefs, M.D., M. Mazen Jamal, M.D., Choon Park, R.N.

University of Texas Southwestern Medical Center, Dallas, TX: (Contract N01-DK-9-2321, Grant M01RR-00633) William M. Lee, M.D., Nicole Crowder, L.V.N., Rivka Elbein, R.N., B.S.N., Patricia Wians, R.N.

University of Southern California, Los Angeles, CA: (Contract N01-DK-9-2325, Grant M01RR-00043) Karen L.

Lindsay, M.D., Carol B. Jones, R.N., Susan L. Milstein, R.N. University of Michigan Medical Center, Ann Arbor, MI: (Contract N01-DK-9-2323, Grant M01RR-00042) Anna S.F. Lok, M.D., Pamela A. Richtmyer, L.P.N., C.C.R.C., Erin Ford, B.S.

Virginia Commonwealth University Health System, Richmond, VA: (Contract N01-DK-9-2322, Grant M01RR00065) Mitchell L. Shiffman, M.D., Richard K. Sterling, M.D., Charlotte Hofmann, R.N., Paula Smith, R.N.

National Institute of Diabetes and Digestive and Kidney Diseases, Liver Disease Section, Bethesda, MD: T. Jake Liang, M.D., Yoon Park, R.N., Elenita Rivera, R.N.
National Institute of Diabetes and Digestive and Kidney Diseases, Division of Digestive Diseases and Nutrition, Bethesda, MD: Leonard B. Seeff, M.D., Patricia R. Robuck, Ph.D., James E. Everhart, M.D., Jay H. Hoofnagle, M.D.

University of Washington, Seattle, WA: (Contract N01DK-9-2318) Chihiro Morishima, M.D., Minjun Chung, B.S., A.S.C.P.

New England Research Institutes, Watertown, MA: (Contract N01-DK-9-2328) Elizabeth C. Wright, Ph.D., Kristin K. Snow, M.Sc., Sc.D., Linda J. Massey

Armed Forces Institute of Pathology, Division of Hepatic Pathology and the Veterans Administration Special Reference Laboratory for Pathology, Washington, DC: Zachary D. Goodman, M.D.

Roche Laboratories, Inc., Nutley, NJ: Raymond S. Koff, M.D., Michael J. Brunda, Ph.D.

Financial relationships of the authors with Hoffmann-La Roche, Inc., are as follows: R.J. Fontana is a consultant and is on the speaker's bureau; S. Mehta is on the speaker's bureau and receives research support; G.T. Everson is a consultant, on the speaker's bureau, and receives research support; J.A. Kahn is on the speaker's bureau, P.F. Malet receives research support; M.Y. Sheikh is on the speaker's bureau and receives research support and R.T. Chung receives research support. In addition, G.T. Everson receives support from Metabolic Solutions and D.R. Gretch receives research support from Bayer. Authors with no financial relationships to disclose are: A.J. Sanyal, B.A. Neuschwander-Tetri, M.C. Doherty, M.G. Ghany and J.E. Everhart.

\section{STUDY HIGHLIGHTS}

\section{What Is Current Knowledge}

- The clinical significance of portal hypertensive gastropathy is unclear due to the lack of large, prospective studies involving well-characterized patients with varying severity of liver disease.

\section{What Is New Here}

- The HALT-C trial demonstrates that $37 \%$ hepatitis C virus (HCV) patients with bridging fibrosis or compensated cirrhosis on liver biopsy have portal hypertensive gastropathy that was severe in only $3 \%$.

- The mucosal mosaic pattern was the most commonly identified upper endoscopic features (33\%) followed by red marks (15\%) and gastric antral vascular ectasia (GAVE) (3\%).

- Both mild and severe portal hypertensive gastropathy were significantly associated with the stage of hepatic fibrosis.

- Patients with portal hypertensive gastropathy were significantly more likely to have esophageal varices compared to subjects without portal hypertensive gastropathy and to have medium/ large varices. However, the mean hemoglobin levels and iron stores were similar 
in patients with and without portal hypertensive gastropathy.

- Independent predictors of portal hypertensive gastropathy include biochemical markers of liver disease severity (serum albumin, bilirubin), portal hypertension (platelet count), insulin resistance (glucose), and non-African American race.

- Contrary to expectations, lifetime smoking and alcohol consumption as well as NSAID use are not associated with mild or severe portal hypertensive gastropathy. However, lifetime smoking and NSAID use in the past year were significantly associated with the presence of GAVE.

Reprint requests and correspondence: Robert J. Fontana, M.D., 3912 Taubman Center, Ann Arbor, MI 48109-0362.

Received July 26, 2005; accepted November 4, 2005.

\section{REFERENCES}

1. Burak KW, Lee SS, Beck PL. Portal hypertensive gastropathy and gastric antral vascular ectasia syndrome. Gut 2001;49:866-72.

2. Thuluvath PJ, Yoo HY. Portal hypertensive gastropathy. Am J Gastroenterol 2002;97:2973-8.

3. Carpinelli C, Primignani M, Preatoni P, et al. Portal hypertensive gastropathy: Reproducibility of a classification, prevalence of elementary lesions, sensitivity and specificity in the diagnosis of cirrhosis of the liver. A NIEC multicenter study. Ital J Gastroenterol 1997;29:533-40.

4. Sarin SK. Diagnostic issues: Portal hypertensive gastropathy and gastric varices. In Portal Hypertension II. Proceedings of the second Baveno International consensus workshop on definitions, methodology and therapeutic strategies. R DeFranchis, ed. Oxford; Blackwell Science, 1996:30-55.

5. Payen JL, Cales P, Voigt JJ, et al. Severe portal hypertensive gastropathy and antral vascular ectasia are distinct entities in patients with cirrhosis. Gastroenterology 1995;108:13444.

6. Merli M, Nicolini G, Angeloni S, et al. The natural history of portal hypertensive gastropathy in patients with liver cirrhosis and mild portal hypertension. Am J Gastroenterol 2004;99:1959-65.

7. Primignani M, Carpinelli L, Preatoni P, et al. Natural history of portal hypertensive gastropathy in patients with liver cirrhosis. Gastroenterology 2000;119:181-7.

8. Gupta R, Saraswat VA, Kumar M, et al. Frequency and factors influencing portal hypertensive gastropathy and duodenopathy in cirrhotic portal hypertension. J Gastro Hepatol 1996;11:728-33.

9. Sarin SK, Shahi HM, Jain M, et al. The natural history of portal hypertensive gastropathy: Influence of variceal eradication. Am J Gastroenterol 2000;95:2888-93.

10. Gostout CJ, Viggiano TR, Balm RK. Acute gastrointestinal bleeding from portal hypertensive gastropathy: Prevalence and clinical features. Am J Gastroenterol 1993;88:20303 .

11. Ohta M, Yamaguchi S, Gotoh N, et al. Pathogenesis of portal hypertensive gastropathy: A clinical and experimental review. Surgery 2002;131:S165-70.
12. Lee WM, Dienstag JL, Lindsay KL, et al. Evolution of the HALT-C trial: Pegylated interferon as a maintenance therapy for chronic hepatitis $\mathrm{C}$ in previous interferon nonresponders. Control Clin Trials 2004;25:472-92.

13. Shiffman ML, Di Bisceglie AM, Lindsay KL, et al. Peginterferon alfa-2a and ribavirin in patients with chronic hepatitis $\mathrm{C}$ who failed prior treatment. Gastroenterology 2004; 126:1015-23.

14. Skinner HA, Sheu WJ. Reliability of alcohol use indices: The lifetime drinking history and the MAST. J Stud Alcohol 1982;43:1157-70.

15. Ishak KG. Chronic hepatitis: Morphology and nomenclature. Mod Pathol 1994;7:690-713.

16. Matthews DR, Hosker JP, Rudenski AS, et al. Homeostasis model assessment: Insulin resistance and beta-cell function from fasting plasma glucose and insulin concentrations in man. Diabetologia 1985;28:412-9.

17. Sanyal AJ, Fontana RJ, Wright E, the Hepatitis C Anti-viral Long-term Treatment Against Cirrhosis (HALT-C) trial investigators. The presence and spectrum of varices in the absence of cirrhosis in patients with chronic hepatitis $\mathrm{C}(\mathrm{Ab}-$ stract). Hepatology 2003;38(suppl 1):218A.

18. Stewart CA, Sanyal AJ. Grading portal gastropathy: Validation of a gastropathy scoring system. Am J Gastroenterol 2003;98:1758-65.

19. Trevino HH, Brady CE, Schenker S. Portal hypertensive gastropathy. Dig Dis 1996;14:258.

20. Ohta M, Yamaguchi S, Gotoh N, et al. Pathogenesis of portal hypertensive gastropathy: A clinical and experimental review. Surgery 2002;131(suppl 1):S165-70.

21. Parikh SS, Desai SB, Prabhu SR, et al. Congestive gastropathy: Factors influencing development, endoscopic findings, Helicobacter pylori infection and microvessel changes. Am J Gastroenterol 1994;89:1036-42.

22. Dore MP, Mura D, Deledda S, et al. Active peptic ulcer disease in patients with hepatitis $C$ virus-related cirrhosis: The role of Helicobacter pylori infection and portal hypertensive gastropathy. Can J Gastroenterol 2004;18:521-4.

23. Merkel C, Schipilliti M, Bighin R, et al. Portal hypertension and portal hypertensive gastropathy in patients with liver cirrhosis: A haemodynamic study. Dig Liver Dis 2003;35:26974.

24. Ito M, Uchida Y, Kamano S, et al. Clinical comparisons between two subsets of gastric antral vascular ectasia. Gastrointest Endosc 2001;53:764.

25. Spahr L, Villeneuve JP, Dufresne MP, et al. Gastric antral vascular ectasia in cirrhotic patients: Absence of relation with portal hypertension. Gut 1999;44:739-42.

26. Auroux J, Lamarque D, Roudot-Thoraval F, et al. Gastroduodenal ulcer and erosions are related to portal hypertensive gastropathy and recent alcohol intake in cirrhotic patients. Dig Dis Sci 2003;48:1118-23.

27. Paradis V, Perlemuter G, Bonvoust F, et al. High glucose and hyperinsulinemia stimulate connective tissue growth factor expression: A potential mechanism involved in progression to fibrosis in non-alcoholic steatohepatitis. Hepatology 2001;34:738-44.

28. Sanyal AJ, Campbell-Sargent C, Mirshahi F, et al. Nonalcoholic steatohepatitis: Association of insulin resistance and mitochondrial abnormalities. Gastroenterology 2001;120:1183-92.

29. Hourigan LF, Macdonald GA, Purdie D, et al. Fibrosis in chronic hepatitis C correlates significantly with body mass index and steatosis. Hepatology 1999;29:12159.

30. Adinolfi LE, Gambardella M, Andreana A, et al. Steatosis accelerates the progression of liver damage of chronic 
hepatitis $\mathrm{C}$ patients and correlates with specific $\mathrm{HCV}$ genotype and visceral obesity. Hepatology 2001;33:1358-64.

31. Uygun A, Kadayifci A, Yesilova Z, et al. Serum leptin levels in patients with non-alcoholic steatohepatitis. Am J Gastroenterol 2000;95:3584-9.

32. Saxena NK, Floyd GJ, Anania FA. Leptin is an independent agonist in the transcriptional activation of the alpha(1) collagen gene in hepatic stellate cells (Abstract). Hepatology 2001;34:359A.

33. Ruhl CE, Everhart JE. Determinants of the association of overweight with elevated serum alanine aminotransferase activity in the United States. Gastroenterology 2003;124:71-9.

34. Weyer C, Funahashi T, Tanaka S, et al. Hypoadiponectinemia in obesity and type 2 diabetes mellitus: Close association with insulin resistance and hyperinsulinemia. J Clin Endocrinol Metab 2001;86:1920-35.

35. Dulai GS, Jensen DM, Kovacs TOG, et al. Endoscopic treatment outcomes in watermelon stomach patients with and without portal hypertension. Endoscopy 2004;36:68-72.

36. Lanza FL. A guideline for the treatment and prevention of NSAID-induced ulcers. Am J Gastroenterol 1998;93:2037.

37. Charneau J, Petit R, Cales P, et al. Antral motility in patients with cirrhosis with or without gastric antral vascular ectasia. Gut 1995;37:488-92.
38. Tanoue K, Tarnawski AS, Kishihiara F, et al. Effect of teprenone on portal hypertensive gastric mucosa. Digestion 1996;57:35-40.

39. Giofre MR, Meduri G, Pallio S, et al. Gastric permeability to sucrose is increased in portal hypertensive gastropathy. Eur J Gastroenterol Hepatol 2000;12:529-33.

40. Schoon IM, Mellstrom D, Oden A, et al. Peptic ulcer disease in older age groups in Gothenburg in 1985: The association with smoking. Age Ageing 1991;20:371.

41. Murata JH, Nogawa AN. Meta-analysis of risk factors for peptic ulcer. Nonsteroidal anti-inflammatory drugs, Helicobacter pylori, and smoking. J Clin Gastroenterol 1997;24:2.

42. Wang CS, Wang ST, Chang TT, et al. Smoking and alanine aminotransferase levels in hepatitis $\mathrm{C}$ virus infection. Arch Intern Med 2002; 162:811-5.

43. Pessione F, Ramond MJ, Njapoum C, et al. Cigarette smoking and hepatic lesions in patients with chronic hepatitis $\mathrm{C}$. Hepatology 2001;34:121-5.

44. Siringo S, Burroughs AK, Bolondi L, et al. Peptic ulcer and its course in cirrhosis an endoscopic and clinical prospective study. J Hepatol 1995;22:633-41.

45. Yoo HY, Eustace JA, Verma S, et al. Accuracy and reliability of the endoscopic classification of portal hypertensive gastropathy. Gastrointest Endosc 2002;56:675-80. 